\title{
Primer consenso transdisciplinario en México sobre cirugía metabólica para el tratamiento de la diabetes tipo 2
}

Palabras clave: Diabetes mellitus, cirugía bariátrica, bypass gástrico, obesidad, consenso, cirugía metabólica.

Key words: Diabetes mellitus, bariatric surgery, gastric bypass, obesity, consensus, metabolic surgery.

* Cirugía Metabólica. Vida Íntegra, Centro para el Control de la Diabetes y Obesidad. Guadalajara, Jalisco. México.

** Professor of Surgery, Chair of Metabolic

\& Bariatric Surgery.

Kings College, London. England.

*** Servicio de Cirugía Bariátrica. Hospital Civil "Fray Antonio Alcalde". Guadalajara, Jalisco.

México.

**** Programa de

Enfermedades Crónico-

Degenerativas, Secretaría de Salud, Jalisco.

***** Professor of

Medicine, Diabetes

\& Obesity Center of

Excellence. University of

Washington, USA.

****** Endocrinología

y Medicina Interna.

Monterrey, Nuevo León.

México.

******* Unidad

Médica de Alta

Especialidad, Hospital

de Especialidades del

Centro Médico de

Occidente del Instituto

Mexicano de Seguro

Social.

Recibido: 03/05/2016

Aceptado: 16/12/2016

\section{First transdisciplinary consensus in Mexico on metabolic surgery for the treatment of type 2 diabetes}

Stefano Sereno-Trabaldo, ${ }^{*}$ Francesco Rubino, ${ }^{* *}$ Rodrigo Prieto-Aldape, ${ }^{* * *}$

Alberto Ocampo-Chavarría, ${ }^{* * * *}$ David M Cummings, ${ }^{* * * * * *}$ Sergio Zúñiga-Guajardo, ${ }^{* * * * * *}$ Alejandro González-Ojeda*******

\section{RESUMEN}

Introducción: Se estima que hay 350 millones de personas con diabetes mellitus, $90 \%$ de las cuales tiene diabetes tipo 2 (DM2); la cirugía gastrointestinal logra altos porcentajes en su control. Objetivos: Revisar la evidencia científica y llegar a un consenso transdisciplinario sobre cirugía metabólica. Métodos: Representantes de sociedades médicas y quirúrgicas, endocrinólogos y cirujanos bariatras de México revisaron la evidencia científica sobre cirugía metabólica. Se votó electrónicamente usando enunciados con respuestas de "Sí" o "No". El consenso se definió por $2 / 3$ de los votos (67\%). Resultados: Un $68 \%$ coincidió en que el tratamiento convencional no es suficiente para lograr un adecuado control de la DM2; 97\% consideró que la cirugía gastrointestinal debe formar parte del tratamiento de la DM2, siendo la derivación gástrica la de mayor elección. Conclusiones: Es el primer consenso en México sobre cirugía metabólica, convocado por la Secretaría de Salud. La cirugía metabólica es parte de los algoritmos de tratamiento de la DM2 internacionalmente. Los participantes mostraron altos grados de consenso sobre terminología, indicaciones, aplicación, necesidad de emitir posicionamientos oficiales y futuro de la cirugía metabólica.

\section{ABSTRACT}

Introduction: It is estimated that around 350 million people have diabetes mellitus; $90 \%$ type 2 diabetes (DM2). The beneficial impact of bariatric surgery in its treatment has been relevant. Objectives: To review the scientific evidence and reach a consensus among endocrinologists and surgeons about metabolic surgery. Material and methods: Representatives from three medical societies and four surgical societies, endocrinologist and bariatric surgeons from Mexico gathered to review the scientific evidence regarding metabolic surgery. Preliminary electronic voting was conducted using statements with responses of "Yes" or "No". Consensus was defined when two-thirds of the votes (67\%) were achieved. Results: Sixty-eight percent of the participants agreed that conventional treatment is not enough to achieve an adequate control of DM2; $97 \%$ considered that gastrointestinal surgery must become part of the treatment of DM2, gastric bypass being the highest choice. Conclusions: It is the first consensus in Mexico on metabolic surgery summoned by the Health Department. Metabolic surgery is part of treatment algorithms for DM2 internationally. All the participants manifested a high degree of consensus on terminology, indications for application, a need to issue official positioning and the future of metabolic surgery.

\section{INTRODUCCIÓN}

a diabetes es un problema de salud mundial. Actualmente se estima que más de 350 millones de personas viven con diabetes, $90 \%$ de ellas con diabetes tipo 2 (DM2). ${ }^{1}$ En México, el número de personas con diabetes se duplicó en las últimas dos décadas. La Encuesta
Nacional de Salud 2012 (ENSANUT) arroja 6.4 millones de mexicanos diagnosticados con diabetes; es decir el 9.2\% de la población. Sin embargo, este número podría ser el doble debido a los casos no diagnosticados. ${ }^{2}$

La diabetes se ha convertido en una verdadera emergencia médica en México: actualmente es la principal causa de muerte 
en hombres y mujeres, y la principal causa de incapacidad, lo que resulta en costos económicos altísimos para quienes la padecen y para el sistema de salud. ${ }^{3,4}$ Es claro que el aumento en la prevalencia de diabetes se asocia con el aumento de la prevalencia de sobrepeso y obesidad, ya que se estima que un $60 \%$ de los diabéticos tiene algún grado de obesidad. ${ }^{5}$ Por otra parte, está bien demostrado que el control estricto de la glucemia reduce la incidencia de las complicaciones microvasculares en la DM2; sin embargo, muy pocos pacientes logran los objetivos de control glucémico, incluso aquéllos que tienen a su disposición múltiples opciones farmacológicas. ${ }^{6,7}$

La inmensa mayoría de los modelos de salud se han enfocado en las modificaciones de hábitos de vida, como la ingesta adecuada de alimentos y la actividad física regular. Pero la prevalencia de obesidad y diabetes sigue aumentando rápidamente. Los medicamentos tradicionales para el control de la diabetes en muchos casos no proveen un adecuado control de la enfermedad. ${ }^{8}$

En un estudio de salud pública, se examinaron varios indicadores para evaluar la atención médica de la diabetes en México. Los resultados mostraron que el $85.6 \%$ de los diabéticos estaban recibiendo hipoglucemiantes orales, y una proporción menos significativa no usaba ningún tratamiento (6.2\%). Pero lo más preocupante es que no hubo diferencia entre los valores de hemoglobina glucosilada (HbA1c) entre el grupo de los individuos que tenían acceso a atención médica y aquéllos que no. El estudio sugiere que el modelo de atención a la diabetes en México es inefectivo y es necesario cambiar de paradigmas. ${ }^{9}$ La epidemia de diabetes debe ser abordada seriamente. Es urgente el desarrollo de programas que reduzcan significativamente el número de nuevos casos. Si no se toman acciones altamente efectivas, en unos pocos años no habrá suficientes recursos para seguir tratando a los sujetos con diabetes y sus complicaciones. ${ }^{10}$

A mediados del siglo XX, apareció nueva información sobre la mejoría de la diabetes después de cirugía gastrointestinal. ${ }^{11,12}$ Estos resultados no generaron mucho interés en la comunidad internacional hasta que el Dr. Walter Pories y su equipo publicaron sus observaciones sobre el extraordinario efecto inmediato en la mejoría de la diabetes que categóricamente presentaban sus pacientes con obesidad y diabetes operados para bajar de peso. ${ }^{13}$ De este modo, la cirugía entró al ámbito médico mundial como una posible opción terapéutica para el control de un problema metabólico tan complejo como la diabetes.

Estudios observacionales y controlados han demostrado que procedimientos quirúrgicos gastrointestinales identificados como "bariátricos" llevan a un control glucémico superior que el tratamiento médico en individuos obesos con DM2, y se ha descrito remisión de la diabetes en rangos del 65 al $83 \%$ de los casos. ${ }^{14-16}$ Además, la cirugía bariátrica parece reducir el riesgo de desarrollar DM2 en los sujetos con obesidad. ${ }^{17}$

Nuevas definiciones se han acuñado para describir los resultados obtenidos en el tratamiento de la diabetes tras cirugía bariátrica, incluso el término de "curación". Sin embargo, no existe ningún consenso al respecto y es preferible utilizar vocablos como "remisión parcial", "remisión completa" y "remisión prolongada". Antes de la cirugía metabólica, no se había siquiera pensado en usar estos términos al referirse a la diabetes. ${ }^{18}$

En la última década, se han realizado múltiples investigaciones médicas, estudios experimentales y trabajos clínicos; se han debatido estos conocimientos en cumbres y congresos mundiales, atrayendo la atención de sociedades y federaciones e, incluso, de hospitales y gobiernos. Hace unos pocos años se acuñó el concepto de "cirugía metabólica". El impacto de la cirugía bariátrica en el control y remisión de la diabetes ha sido tan relevante que estos procedimientos gastrointestinales han sido incluidos en los algoritmos para el tratamiento de la diabetes por parte de sociedades médicas como la Asociación Americana de Diabetes (ADA) y la International Diabetes Federation (IDF),$^{19}$ entre otras. Recientemente, se publicó el posicionamiento conjunto por parte de 45 organizaciones internacionales con respecto a la inclusión de la cirugía metabólica en el algoritmo de tratamiento de la diabetes tipo 2. De estas organizaciones, 30 son médicas (diabetes, endocrinología y gastroenterología) y 15 , quirúrgicas. En dicho trabajo, con base en la evidencia científica, se consensó que la cirugía metabólica debe ser recomendada 
para tratar la DM2 en personas con obesidad grado III y en aquéllas con obesidad grado II cuando la hiperglucemia es inadecuadamente controlada con cambios de hábitos de vida y terapia médica óptima. ${ }^{20}$

Adicionalmente, la cirugía metabólica ha mostrado efectos positivos en el control de otras comorbilidades asociadas con la obesidad como hipertensión, dislipidemia, síndrome metabóliCo, enfermedad renal crónica, hipertrofia ventricular, esteatohepatitis no alcohólica y apnea obstructiva del sueño. ${ }^{21}$ La cirugía metabólica, como cualquier otra cirugía, conlleva morbimortalidad, pero es tan baja que logra ofrecerse con una gran seguridad cuando se realiza en centros hospitalarios y con equipos quirúrgicos certificados. La mortalidad perioperatoria de la cirugía bariátrica oscila entre 0.10 y $0.35 \% .^{22,23}$

Sin embargo, a pesar de toda esta evidencia y de que existen recomendaciones y guías clínicas que indican el uso de la cirugía gastrointestinal para pacientes con DM2, muy pocos de estos individuos tienen acceso a la cirugía. México es uno de los países con mayor número de personas con diabetes en el mundo y, paradójicamente, la investigación en cirugía metabólica es muy pobre. ${ }^{24}$

Está claro que la cirugía no es la respuesta mágica ni la panacea para dar solución a un problema tan grave y complejo como la DM2, pero es de mayor trascendencia e importancia que la comunidad médica y científica identifique con claridad cuáles sujetos son los que más se pueden beneficiar de ser sometidos a cada tipo de cirugía metabólica, y que en la medida en que este conocimiento avance, se pueda comprender mejor la patofisiología de la diabetes para desarrollar mejores tratamientos que los que tenemos hoy en día.

\section{Objetivos}

Con este concepto bien identificado, la Secretaría de Salud de Jalisco convocó a las principales asociaciones médicas y quirúrgicas de México relacionadas con la diabetes y la obesidad, así como a expertos endocrinólogos y cirujanos bariatras, con tres objetivos:

1. Generar un diálogo científico entre clínicos (endocrinólogos, diabetólogos, investigado- res) y quirúrgicos (principalmente cirujanos bariatras) basados en la revisión de la evidencia sobre cirugía y diabetes tipo 2.

2. Crear una fuerza de tarea para el estudio de la cirugía metabólica en México, para colaborar con su contraparte, la fuerza de tarea internacional para el estudio de la cirugía de diabetes.

3. Generar un posicionamiento con respecto a la cirugía metabólica y la diabetes tipo 2 en personas con obesidad.

\section{MATERIAL Y MÉTODOS}

Convocados por la Secretaría de Salud de Jalisco, durante dos días los representantes de tres sociedades médicas y cuatro sociedades quirúrgicas de México se reunieron junto con un grupo de expertos clínicos y cirujanos para revisar la mejor evidencia científica disponible a la fecha, sobre cirugía metabólica.

\section{Participantes}

- Asociaciones médicas:

- Colegio de Cardiólogos de Jalisco.

- Sociedad Jalisciense de Endocrinología y Nutrición.

- Sociedad Mexicana de Nutrición y Endocrinología.

- Asociaciones quirúrgicas:

- Asociación Mexicana de Cirugía Endoscópica.

- Asociación Mexicana de Cirugía General.

- Colegio Mexicano para la Cirugía de Obesidad y Enfermedades Metabólicas.

- Colegio de Especialistas en Cirugía General de Jalisco.

$$
\text { Cirujanos } \mathrm{n}=17 \quad \text { No cirujanos } \mathrm{n}=7
$$

En el grupo de cirujanos, todos fueron especialistas en cirugía bariátrica. En el grupo de no cirujanos hubo cinco endocrinólogos, un cardiólogo y un epidemiólogo encargado del programa de diabetes de la Secretaría de Salud de Jalisco.

El programa incluyó diferentes módulos: la epidemiología de la diabetes y obesidad, costo-beneficio del control de la diabetes, tratamientos médicos y quirúrgicos actuales para el 
tratamiento de la diabetes y sus resultados, ponerse de acuerdo en la terminología, métodos experimentales, teorías sobre los mecanismos de control de la diabetes con la cirugía, impacto en el riesgo cardiovascular comparando los tratamientos médicos con los quirúrgicos en el abordaje de la obesidad y diabetes, prioridades en la investigación, centros de excelencia para realizar cirugía bariátrica y metabólica, creación de una fuerza de tarea para el estudio de la cirugía metabólica en México, revisión de las guías internacionales que mencionan el uso de la cirugía metabólica y el impacto de los posicionamientos en las políticas de salud.

Antes de iniciar cada módulo, se realizó un voto electrónico preliminar por parte de los expertos y los representantes de las sociedades usando enunciados que llevaron a respuestas sencillas de "Sí o "No". Después del desarrollo del módulo y la discusión del tema, se volvió a votar electrónicamente usando los mismos enunciados que en el voto preliminar.

El consenso se definió cuando se alcanzaron dos tercios de los votos; es decir, cuando se estuvo de acuerdo en el $67 \%$ de los votos. Los grados de consenso se dividieron en tres grupos: A: 90 a 100\%, B: 78 a 89\% y C: 67 a 77\%.

Todas las conferencias y momentos de votación fueron grabadas en video y transmitieron en vivo vía internet, y pueden revisarse en el siguiente enlace: http://new.livestream.com/ accounts/1568456/events/1865307

\section{RESULTADOS}

Entre los resultados más relevantes del consenso entre médicos clínicos y quirúrgicos se encuentran los siguientes. Un 68\% de los participantes coincidió en que el control de la diabetes tipo 2 en México no es adecuado con los tratamientos convencionales actuales ( $43 \%$ de los cirujanos y $75 \%$ de los no cirujanos); $90 \%$ de los participantes consideró que el control metabólico por medio de $\mathrm{HbA} 1 \mathrm{c}$ es malo en la mayoría de los pacientes ( $100 \%$ de los no cirujanos y $88 \%$ de los cirujanos).

Un 93\% (94\% de los cirujanos y $80 \%$ de los no cirujanos) opinó que la cirugía gastrointestinal puede ser apropiada para el tratamiento de la DM2 en individuos que son candidatos quirúrgicos elegibles con índice de masa corporal (IMC) 30-35 y que son controlados inadecuadamente por estilo de vida y tratamiento médico; 91\% (92\% de los cirujanos y $100 \%$ de los no cirujanos) coincidieron en que sujetos con IMC > 35 que son candidatos y que son sometidos a cirugía gastrointestinal (derivación gástrica, gástrica anillada, derivación biliopancreática, manga) tienen una tasa mayor de supervivencia, con disminución del riesgo cardiovascular, comparados con las personas que no son operadas. El 100\% considera que los procedimientos de derivación intestinal (derivación gástrica y DBP) mejoran la diabetes por medio de mecanismos diferentes a los cambios alimenticios y pérdida de peso.

El 100\% considera que debe haber investigaciones y ensayos clínicos controlados para determinar el uso apropiado de la cirugía gastrointestinal en pacientes diabéticos con un IMC < 35, así como la necesidad de crear una fuerza de trabajo en México conformada por expertos cirujanos y no cirujanos para el estudio de la misma. El 100\% refirió interés en estudiar la cirugía metabólica y $80 \%$ dijo tener suficientes elementos para crear un posicionamiento sobre el tema tratado.

Los resultados completos se encuentran anexos en el documento Resultados del primer consenso en México sobre cirugía metabólica.

\section{DISCUSIÓN}

Ésta es la primera vez que las principales asociaciones, sociedades y colegios médicos y quirúrgicos relacionados con diabetes y obesidad, junto con un grupo de expertos en la materia, se reunieron convocados por una secretaría de salud para discutir y evaluar la cirugía metabólica.

Desde que se identificó que la cirugía gastrointestinal tiene un efecto terapéutico benéfico en los individuos con obesidad y diabetes, ha habido reuniones para discutir sobre la cirugía metabólica en México, ${ }^{25}$ pero hasta donde tenemos conocimiento, ésta fue la primera vez en que ambos, endocrinólogos y cirujanos, revisaron, discutieron y votaron de manera conjunta para lograr un consenso sobre este tema tan trascendente.

Este hecho debe quedar bien subrayado porque el estudio de la cirugía metabólica 
quedaría incompleto si uno de los dos, clínicos o quirúrgicos, no estuviera presente.

La información fue convincente y contundente cuando se habló de los altos porcentajes de control de diabetes tras la cirugía gastrointestinal y su impacto favorable en la disminución del riesgo cardiovascular.

Los resultados obtenidos en este foro claramente mostraron que el punto de vista y el conocimiento de los endocrinólogos influenciaron, enriquecieron y modificaron la opinión de los cirujanos en ciertos tópicos, y viceversa.

Los participantes estuvieron de acuerdo en que el control glucémico intensivo y el tratamiento multiobjetivo para la diabetes tipo 2 es costo-efectivo, pero que su control en México con tratamientos convencionales es pobre e inadecuado para la mayoría de los sujetos.

También se encontró un alto grado de consenso entre los participantes al definir cirugía metabólica como una cirugía gastrointestinal (derivación, banda, manga o DBP) que se realiza con la intención de mejorar y/o controlar la diabetes mellitus tipo 2 y otros componentes del síndrome metabólico, y que debe ser incluida en los algoritmos para el tratamiento de personas con diabetes y un IMC $\geq 35$ que se controlan de manera inadecuada con cambios de hábitos de vida y la mejor terapia médica disponible.

Clínicos y cirujanos compartieron un alto grado de consenso en el hecho de que la cirugía debe ser considerada para el tratamiento de la diabetes tipo 2 en pacientes que son candidatos a cirugía que tengan un IMC igual o mayor a 35 , o un IMC de 30 a 35 que no estén adecuadamente controlados pese a cambios de estilo de vida y el mejor tratamiento médico disponible; la derivación gástrica fue la única técnica quirúrgica aceptada al compararla con la banda gástrica, la gastrectomía en manga o las derivaciones biliopancreáticas (DBP).

Los expertos manifestaron un alto consenso en que los nuevos procedimientos quirúrgicos (experimentales) no deben realizarse fuera de protocolos de investigación aprobados por comités éticos.

Hubo un alto grado de consenso con respecto a la terminología, siendo la intención del tratamiento lo que le da el nombre a la cirugía: si la prioridad es tratar obesidad, en- tonces la cirugía deberá llamarse "bariátrica"; si la intención principal es controlar la diabetes (en un individuo con obesidad), entonces los procedimientos deberán llamarse "cirugía para diabetes"; y cuando el propósito es controlar el síndrome metabólico, entonces estaremos hablando de "cirugía metabólica".

La Secretaría de Salud de Jalisco desarrolló un papel muy importante porque, siendo la institución con mayor calidad moral como autoridad, encontró un eco en todas las sociedades médicas y quirúrgicas que participaron en este foro con el mejor ánimo de objetividad e imparcialidad.

Todas las sociedades médicas y quirúrgicas se comprometieron para crear su propio posicionamiento con respecto a la cirugía metabólica.

La diabetes y la obesidad son un problema de salud en México. Actualmente, el modelo de atención médica para la diabetes en México es inefectivo, pero la cirugía gastrointestinal ha mostrado mejores porcentajes de control de la diabetes tipo 2 y la obesidad en el largo plazo.

La cirugía metabólica debe ser estudiada por endocrinólogos y cirujanos para crear las mejores recomendaciones. Las sociedades médicas y quirúrgicas en México mostraron estar dispuestas a elaborar su propio posicionamiento sobre la cirugía metabólica, y la Secretaría de Salud de Jalisco se dejó ver como el mejor mediador para facilitar el diálogo y la comprensión entre ellas.

Se espera que en futuras reuniones como este II Foro se cree una fuerza de tarea mexicana para el estudio de la cirugía metabólica en México que ayude a avanzar en la creación de mejores tratamientos para las personas con obesidad y diabetes.

\section{Agradecimientos (participantes)}

Jesús Argüelles-Sarmiento, Ricardo Cohen, J Miguel Escalante-Pulido, Jorge EspinozaCampos, Jorge González-Estrada, Miguel F Herrera, Juan Antonio López-Corvalá, Enrique Luque de León, Eduardo Márquez-Rodríguez, José Antonio Muñoz-Serrano, Fernando Ortiz-Galván, Ariel Ortiz-Lagarde, Juan Pablo Pantoja-Millán, Gilberto Ungson-Beltrán, Pablo Zorrilla-Blanco. 


\section{Créditos}

El diseño de la propuesta fue realizado por los autores SST, RPA y AGO. El diseño del instrumento de evaluación fue realizado por FR y DMC. Los convocadores de la Secretaría de Salud Jalisco, AOE, y Monterrey, SZG. El manuscrito fue redactado por SST, RPA, AGO y se realizó una revisión crítica por parte de FR y DMC. Todos los autores coincidieron en la aprobación final del manuscrito.

\section{Conflicto de intereses}

Los autores declaran que no existe ningún conflicto de intereses económico o de cualquier otro tipo.

\section{REFERENCIAS}

1. World Health Organization. Diabetes Fact Sheet No. 312. August 2011. Available from: http://www.who.int/

2. Gutiérrez JP, Rivera-Dommarco J, Shamah-Levy T, Villalpando-Hernández S, Franco A, Cuevas-Nasu L, et al. National Survey of Health and Nutrition 2012. National results. Cuernavaca, Mexico: National Institute of Health; 2012.

3. Aguilar-Salinas CA. Promoting health for the prevention of chronic degenerative diseases linked to diet and lifestyle. In: Mariano García Viveros, editor. Community Health and promotion of health. ICEPSS Publishers; 1999. ISBN 84-89151-17-2. Spain.

4. Ministry of Health. Directorate General of Information for Health. CONAPO, 2002. Projections of Population of Mexico, 2000-2050.

5. Kramer H, Cao G, Dugas L, Luke A, Cooper R, DurazoArvizu R. Increasing BMI and waist circumference and Prevalence of obesity Among adults with type 2 diabetes: the National Health and Nutrition Examination Surveys. J Diabetes Complications. 2010; 24: 368-374.

6. Shaya FT, Yan X, Lin PJ, Simoni-Wastila L, Bron M, Baran $\mathrm{R}$, et al. U.S. trends in glycemic control treatment, and comorbidity burden in patients with diabetes. J Clin Hypertens (Greenwich). 2010; 12: 826-832.

7. Gaede P, Vedel P, Larsen N, Jensen GV, Parving HH, Pedersen O. Multifactorial intervention and cardiovascular disease in patients with type 2 diabetes. N Engl J Med. 2003; 348: 383-393.

8. U.K. prospective diabetes study 16. Overview of 6 years' therapy of type II diabetes: a progressive disease. U.K. Prospective Diabetes Study Group. Diabetes. 1995; 44: 1249-1258.

9. González-Villalpando C, López-Ridaura R, Campuzano JC, González-Villalpando ME. The status of diabetes care in Mexican population: are we making a difference? Results of the National Health and Nutrition Survey 2006. Salud Publica Mex. 2010; 52 Suppl 1: S36-S43.

10. Aguilar-Salinas CA, Gómez-Pérez FJ. The "Declaración de Acapulco": a proposal to reduce incidence of diabetes in Mexico. Rev Invest Clin. 2006; 58: 71-77.
11. Friedman MN, Sancetta AJ, Magovern GJ. The amelioration of diabetes mellitus following subtotal gastrectomy. Surg Gynecol Obstet. 1955; 100: 201-204.

12. Forgács $S$, Halmos T. Improvement of glucose tolerance in diabetics following gastrectomy. Z Gastroenterol. 1973; 11: 293-296.

13. Pories WJ, Swanson MS, MacDonald KG, Long SB, Morris PG, Brown BM, et al. Who would have thought it? An operation proves to be the most effective therapy for adult-onset diabetes mellitus. Ann Surg. 1995; 222: 339-350; discussion 350-352.

14. Buchwald H, Estok R, Fahrbach K, Banel D, Jensen $M D$, Porisk WJ, et al. Weight and type 2 diabetes after bariatric surgery: systematic review and meta-analysis. Am J Med. 2009; 122: 248-256.

15. Mingrone G, Panunzi S, De Gaetano A, Guidone C, laconelli A, Leccesi L, Nanni et al. Bariatric surgery versus conventional medical therapy for type 2 diabetes. N Engl J Med. 2012; 366: 1577-1585.

16. Schauer PR, Kashyap SR, Wolski K, Brethauer SA, Kirwan JP, Pothier CE, et al. Bariatric surgery versus intensive medical therapy in obese patients with diabetes. N Engl J Med. 2012; 366: 1567-1576.

17. Carlsson LM, Peltonen M, Ahlin S, Anveden $\AA$, Bouchard C, Carlsson B, et al. Bariatric surgery and prevention of type 2 diabetes in Swedish obese subjects. N Engl J Med. 2012; 367: 695-704.

18. González VC, Stern AM, Arredondo PB, Martínez DS. The level of metabolic monitoring in low income Mexico City diabetics. The Mexico City diabetes study. Arch Med Res. 1994; 25: 387-392.

19. American Diabetes Association. Standards of medical care in diabetes-2013. Diabetes Care. 2013; 36 Suppl 1: S11-S66.

20. Rubino F, Nathan DM, Eckel RH, Schauer PR, Alberti $\mathrm{KG}$, Zimmet PZ, et al. Metabolic surgery in the treatment algorithm for type 2 diabetes: a joint statement by International Diabetes Organizations. Diabetes Care. 2016; 39: 861-877.

21. Buchwald H, Avidor Y, Braunwald E, Jensen MD, Pories W, Fahrbach K, et al. Bariatric surgery: a systematic review and meta-analysis. JAMA. 2004; 292: 1724-1737.

22. Athyros VG, Tziomalos K, Karagiannis A, Mikhailidis DP. Cardiovascular benefits of bariatric surgery in morbidly obese patients. Obes Rev. 2011; 12: 515-524.

23. Buchwald H, Estok R, Fahrbach K, Banel D, Sledge I. Trends in mortality in bariatric surgery: a systematic review and meta-analysis. Surgery. 2007; 142: 621632, discussion 632-635.

24. Romero-Mejía C. Metabolic surgery in Latin America. Cir Gen. 2010; 32: 121-124.

25. Sereno-Trabaldo S, Cote-Estrada L. Resultados de la Encuesta del I Foro para el Estudio de la Cirugía Metabólica en México. Cir Gen. 2010; 32: 90-95.
Correspondencia:
Dr. Stefano Sereno-Trabaldo
Vida Íntegra, Centro para el Control
de la Diabetes y Obesidad.
Calle Québec Núm. 631, interior 312,
Col. Prados de Providencia, 44670,
Guadalajara, Jalisco, México.
Tel: (33) 36404244 y 45
E-mail: ssereno@gmail.com 
ANEXO.

RESULTADOS DEL PRIMER CONSENSO EN MÉXICO SOBRE CIRUGÍA METABÓLICA

\section{MÓDULO 1}

Resultados clínicos sobre el tratamiento convencional para diabetes tipo 2 en México.

\section{1. ¿Tratar médicamente la diabetes tipo 2 es costo-efectivo? Sí \\ Consenso: Alto}

Antes de la presentación:

Votación total: Sí $71 \% \quad$ No $29 \%$

Cirujanos: $\quad$ Sí 50\% No 50\%

No cirujanos: Sí 100\%

Después de la presentación:

Votación total: Sí $90 \% \quad$ No $10 \%$

Cirujanos: $\quad$ Sí $89 \%$ No $11 \%$

No cirujanos: Sí 100\%

Comentario: Después de la presentación de los endocrinólogos, los cirujanos incrementaron su opinión sobre la costo-efectividad para tratar la diabetes tipo 2.

\section{El control glucémico intensivo, ies costo-efectivo? Sí \\ Consenso: Alto}

Antes de la presentación:

Votación total: Sí $86 \% \quad$ No $14 \%$

Cirujanos: $\quad$ Sí $73 \%$ No $27 \%$

No cirujanos: Sí 100\%

Después de la presentación:

Votación total: Sí $90 \% \quad$ No $10 \%$

Cirujanos: $\quad$ Sí $85 \%$ No $15 \%$

No cirujanos: Sí 100\%

Comentario: Después de la presentación de los endocrinólogos, los cirujanos aumentaron su opinión sobre la costo-efectividad del control glucémico intensivo en la diabetes tipo 2.

\section{El tratamiento multiobjetivo en la diabetes} tipo 2, ies costo-efectivo? Sí

Consenso: Alto

Antes de la presentación:

Votación total: Sí 95\% No 5\%

Cirujanos: $\quad$ Sí $91 \%$ No 9\%

No cirujanos: Sí 100\%

Después de la presentación:

Votación total: Sí 95\% No 5\%

Cirujanos: $\quad$ Sí $91 \%$ No 9\%

No cirujanos: Sí 100\%
4. El control de la diabetes mellitus tipo 2 en México, ies adecuado con los tratamientos convencionales actuales? No

\section{Consenso: Alto}

Antes de la presentación:

Votación total: Sí 12\% No 88\%

Cirujanos: $\quad$ Sí 25\% No $75 \%$

No cirujanos: $\quad$ Sí $0 \% \quad$ No $100 \%$

Después de la presentación:

Votación total: Sí 32\% No 68\%

Cirujanos: $\quad$ Sí $57 \%$ No $43 \%$

No cirujanos: Sí 25\% No $75 \%$

Comentario: Después de la presentación, cirujanos y no cirujanos aumentaron su opinión sobre la adecuación del control de la DM2 en México con el tratamiento convencional, pero no existe consenso sobre ello. De manera interesante, antes de la presentación, los no cirujanos tenían un consenso fuerte de que no hay adecuado control de la DM con el tratamiento convencional, y después de la presentación, 25\% cambiaron su opinión. Un 32\% de los cirujanos también cambiaron su opinión.

5. El control metabólico (HbA1c), ies malo en la mayoría de los sujetos con DM2? Sí Consenso: Medio

Antes de la presentación:

Votación total: $\quad$ Sí el 85\% No 15\%

Cirujanos: $\quad$ Sí $67 \%$ No $33 \%$

No cirujanos: Sí 100\% No 0\%

Después de la presentación:

Votación total: Sí 90\% No 10\%

Cirujanos: $\quad$ Sí $88 \% \quad$ No $12 \%$

No cirujanos: Sí 100\% No 0\%

Comentario: Después de la presentación, los cirujanos se dieron cuenta de que el control metabólico medido con HbA1c es malo.

6. ¿La historia natural de la diabetes tipo 2 (disfunción de la célula Beta) se ha modificado con la terapéutica farmacológica actual? Sí Consenso: Bajo

Antes de la presentación:

Votación total: Sí 59\% No 41\%

Cirujanos: $\quad$ Sí $50 \%$ No 50\%

No cirujanos: Sí 100\%

Después de la presentación: 
$\begin{array}{lll}\text { Votación total: } & \text { Sí } 73 \% & \text { No } 27 \% \\ \text { Cirujanos: } & \text { Sí } 70 \% & \text { No 30\% } \\ \text { No cirujanos: } & \text { Sí } 75 \% & \text { No } 25 \%\end{array}$

Comentario: Antes de las presentaciones, las opiniones de los cirujanos y los no cirujanos eran diferentes. Todos los no cirujanos pensaron que la historia natural de la DM2 se ha modificado con la terapéutica farmacológica actual y los cirujanos tuvieron opiniones divididas. Después de la presentación, ambos, 2/3 de los cirujanos y no cirujanos acordaron que la historia natural de la DM2 sí fue modificada con los medicamentos. Este hecho nos hace pensar si tuvimos la mejor evidencia disponible.

\section{MÓDULO 2}

Resultados clínicos de los tratamientos quirúrgicos para diabetes tipo 2 en México.

7. La cirugía gastrointestinal debe considerarse para el tratamiento de la diabetes tipo 2 en personas que son candidatas quirúrgicas elegibles con IMC $\geq 35$ que son controladas inadecuadamente por estilo de vida y tratamiento médico. Sí

Consenso: Alto

Antes de la presentación:

Votación total: Sí: $90 \% \quad$ No $10 \%$

Cirujanos: $\quad$ Sí $91 \%$ No $9 \%$

No cirujanos: Sí 67\% No 33\%

Después de la presentación:

Votación total: Sí $97 \%$ No 3\%

Cirujanos: $\quad$ Sí 100\% No 0\%

No cirujanos: Sí 95\% No 5\%

Comentario: Los no cirujanos no consideraron

la cirugía en estas condiciones antes de la presentación. Después de la presentación, hasta 95\% estuvo de acuerdo en la cirugía.

8. La derivación gástrica debe considerarse para el tratamiento de la diabetes tipo 2 en pacientes que son candidatos quirúrgicos elegibles con IMC $\geq 35$ que son controlados inadecuadamente por estilo de vida y tratamiento médico. Sí

Consenso: Alto

Antes de la presentación:

Votación total: Sí 100\% No 0\%

Cirujanos: $\quad$ Sí $100 \%$ No 0\%

No cirujanos: Sí 100\% No 0\%

Después de la presentación: $\begin{array}{lll}\text { Votación total: } & \text { Sí 100\% } & \text { No 0\% } \\ \text { Cirujanos: } & \text { Sí 100\% } & \text { No 0\% } \\ \text { No cirujanos: } & \text { Sí 100\% } & \text { No 0\% }\end{array}$

9. La banda gástrica ajustable debe considerarse para el tratamiento de la diabetes tipo 2 en individuos que son candidatos quirúrgicos elegibles con IMC $\geq 35$ que son controlados inadecuadamente por estilo de vida y tratamiento médico. No

Consenso: Alto

Antes de la presentación:

Votación total: Sí 5\% No 95\%

Cirujanos: $\quad$ Sí $0 \% \quad$ No $100 \%$

No cirujanos: Sí 33\% No 67\%

Después de la presentación:
Votación total: $\quad$ Sí 7\% $\quad$ No $93 \%$

Cirujanos: $\quad$ Sí 0\% $\quad$ No $100 \%$

No cirujanos: Sí 50\% No 50\%

Comentario: Después de la presentación, los no cirujanos fueron más propensos a considerar la banda como una opción para el control metabólico. Los cirujanos nunca la consideraron, pero cuando se les preguntó por qué, no hubo comentarios al respecto.

10. La manga gástrica debe considerarse para el tratamiento de la diabetes tipo 2 en sujetos que son candidatos quirúrgicos elegibles con IMC $\geq 35$ que son controlados inadecuadamente por estilo de vida y tratamiento médico. Sí

Consenso: No

Antes de la presentación:

Votación total: Sí: 50\% No 50\%

Cirujanos: $\quad$ Sí $67 \%$ No $33 \%$

No cirujanos: Sí 0\% No $100 \%$

Después de la presentación:

Votación total: Sí: 50\% No 50\%

Cirujanos: $\quad$ Sí $47 \%$ No $53 \%$

No cirujanos: Sí $40 \% \quad$ No $60 \%$

Comentario: Después de la presentación, los cirujanos se mostraron más prudentes para usar una gastrectomía en manga para fines metabólicos. Por otro lado, los no cirujanos, que antes de la presentación nunca habrían considerado esta opción, cambiaron y la considerarían en el $40 \%$. Aunque no hubo consenso, esto es un tema que sería objeto de discusión en el futuro.

11. Las derivaciones biliopancreáticas (DBP) deben considerarse para el tratamiento de la diabetes tipo 2 en personas que son candida- 
tas quirúrgicos elegibles con $\mathrm{IMC} \geq 35$ que son controladas inadecuadamente por estilo de vida y tratamiento médico. Sí

Consenso: Bajo

Antes de la presentación:

$\begin{array}{lll}\text { Votación total: } & \text { Sí 38\% } & \text { No } 62 \% \\ \text { Cirujanos: } & \text { Sí } 50 \% & \text { No } 50 \% \\ \text { No cirujanos: } & \text { Sí 0\% } & \text { No } 100 \%\end{array}$

Después de la presentación:

Votación total: Sí $68 \%$ No $32 \%$

Cirujanos: $\quad$ Sí $75 \%$ No $25 \%$

No cirujanos: Sí 40\% No $60 \%$

Comentario: Después de la presentación, los cirujanos considerarían mejor hacer un BPD con fines metabólicos. Por otro lado, los no cirujanos, que antes de la presentación nunca habrían considerado esta opción, la tomarían en cuenta en el $40 \%$.

12. La cirugía gastrointestinal puede ser apropiada para el tratamiento de la diabetes tipo 2 en pacientes que son candidatos quirúrgicos elegibles con IMC 30-35 que son controlados inadecuadamente por estilo de vida y tratamiento médico. Sí

Consenso: Alto

Antes de la presentación:

Votación total: Sí 95\% No 5\%

Cirujanos: $\quad$ Sí 100\% No 0\%

No cirujanos: Sí $75 \%$ No $25 \%$

Después de la presentación:

Votación total: Sí 93\% No 7\%

Cirujanos: $\quad$ Sí $94 \%$ No 6\%

No cirujanos: $\quad$ Sí $80 \% \quad$ No $20 \%$

13. La derivación gástrica puede ser apropiada para el tratamiento de la diabetes tipo 2 en individuos que son candidatos quirúrgicos elegibles con IMC 30-35 que son controlados inadecuadamente por estilo de vida y tratamiento médico. Sí

Consenso: Alto

Antes de la presentación:

Votación total: Sí $77 \%$

Cirujanos: $\quad$ Sí $90 \%$ No $10 \%$

No cirujanos: Sí 100\% No 0\%

Después de la presentación:

Votación total: Sí 93\% No 7\%

Cirujanos: $\quad$ Sí $94 \%$ No 6\%

No cirujanos: Sí 100\% No 0\%

Comentario: De manera interesante, los no

cirujanos considerarían más que los cirujanos que la derivación gástrica puede ser apropiada para tratar la DM2 en sujetos con IMC 30-35 no controlados.

14. La banda gástrica ajustable puede ser apropiada para el tratamiento de la diabetes tipo 2 en personas que son candidatas quirúrgicas elegibles con IMC 30-35 que son controladas inadecuadamente por estilo de vida y tratamiento médico. No

Consenso: Alto

Antes de la presentación:

Votación total: $\quad$ Sí 8\% $\quad$ No $92 \%$

Cirujanos: $\quad$ Sí 0\% $\quad$ No 100\%

No cirujanos: Sí 50\% No 50\%

Después de la presentación:

Votación total: Sí 3\% No 97\%

Cirujanos: $\quad$ Sí $100 \%$ No $0 \%$

No cirujanos: Sí $20 \% \quad$ No $80 \%$

Comentario: Después de la presentación, los no cirujanos cambiaron de opinión y aumentaron su reticencia a usar banda gástrica como tratamiento de DM2 de difícil control en IMC 30-35.

15. La gastrectomía en manga puede ser apropiada para el tratamiento de la diabetes tipo 2 en pacientes que son candidatos quirúrgicos elegibles con IMC 30-35 que son controlados inadecuadamente por estilo de vida y tratamiento médico. Sí

Consenso: No

Antes de la presentación:

$$
\begin{array}{lll}
\text { Votación total: } & \text { Sí 62\% } & \text { No } 38 \% \\
\text { Cirujanos: } & \text { Sí 60\% } & \text { No } 40 \% \\
\text { No cirujanos: } & \text { Sí 0\% } & \text { No } 100 \%
\end{array}
$$

Después de la presentación:

Votación total: Sí 59\% No 41\%

Cirujanos: $\quad$ Sí 59\% No 41\%

No cirujanos: Sí 50\% No 50\%

Comentario: Después de la presentación, los no cirujanos cambiaron de 0 a 50\% su aceptación hacia la manga para el tratamiento de DM2 de difícil control en IMC 30-35, pero no se logró llegar a un consenso.

16. La DBP puede ser apropiada para el tratamiento de la diabetes tipo 2 en individuos que son candidatos quirúrgicos elegibles con IMC 30-35 que son controlados inadecuadamente por estilo de vida y tratamiento médico. No

Consenso: Bajo

Antes de la presentación: 
Votación total: Sí 22\%

Cirujanos: $\quad$ Sí 25\%

No cirujanos: $\quad$ Sí 0\%

Después de la presentación:

Votación total: Sí: 31\%

Cirujanos: $\quad$ Sí $31 \%$

Comentario: Después de la presentación, los cirujanos disminuyeron un poco su opinión a favor de la DBP para tratar la DM2 de difícil control en IMC 30-35, pero los no cirujanos aumentaron su aceptación.

17. La cirugía bariátrica es segura cuando es realizada por cirujanos certificados que trabajan con equipos multidisciplinarios en hospitales certificados. Sí

Consenso: Alto

Antes de la presentación:

$$
\begin{array}{lll}
\text { Votación total: } & \text { Sí 100\% } & \text { No 0\% } \\
\text { Cirujanos: } & \text { Sí 100\% } & \text { No 0\% } \\
\text { No cirujanos: } & \text { Sí 100\% } & \text { No 0\% }
\end{array}
$$

Después de la presentación:

Votación total: Sí 100\% No 0\%

Cirujanos: $\quad$ Sí 100\% No 0\%

No cirujanos: Sí $100 \%$ No 0\%

18. La cirugía gastrointestinal (derivación gástrica, banda gástrica, BPD, manga), realizada con la intención de mejorar y/o controlar la DM tipo 2 y otros componentes del síndrome metabólico, debe ser Ilamada "cirugía metabólica". Sí

Consenso: Alto

Antes de la presentación:

$\begin{array}{lll}\text { Votación total: } & \text { Sí } 77 \% & \text { No } 23 \% \\ \text { Cirujanos: } & \text { Sí } 67 \% & \text { No } 33 \% \\ \text { No cirujanos: } & \text { Sí } 50 \% & \text { No } 50 \%\end{array}$

Después de la presentación:

Votación total: Sí 92\% No 8\%

Cirujanos: $\quad$ Sí $100 \%$ No 0\%

No cirujanos: Sí $60 \% \quad$ No $40 \%$

Comentario: Gran impacto de la presentación, ya que incrementó sensiblemente el consenso sobre esta terminología entre cirujanos y aumentó el acuerdo de los no cirujanos, con lo que se llegó casi al consenso.

19. La cirugía gastrointestinal (derivación gástrica, banda gástrica, BPD, manga) se debe incluir en los algoritmos para el tratamiento de sujetos diabéticos con un IMC $\geq 35$ $\mathrm{kg} / \mathrm{m}^{2}$ que son controlados inadecuadamente por estilo de vida y tratamiento médico. Sí Consenso: Alto

Antes de la presentación:

Votación total: Sí 81\% No 19\%

Cirujanos: $\quad$ Sí $85 \%$ No $15 \%$

No cirujanos: Sí $60 \%$ No $40 \%$

Después de la presentación:

Votación total: Sí 96\% No 4\%

Cirujanos: $\quad$ Sí 93\% $\quad$ No $7 \%$

No cirujanos: Sí 100\% No 0\%

Comentario: Alto impacto de la presentación, principalmente entre los no cirujanos, que están dispuestos a incluir la cirugía Gl bajo estas condiciones en los algoritmos de tratamiento de diabetes.

\section{MÓDULO 3}

Reducción en el riesgo CV y la mortalidad en personas con diabetes tipo 2. Cirugías no convencionales para el control metabólico.

20. Nuevos procedimientos como la exclusión duodenal, transposición ileal y procedimientos intraluminales, que muestran resultados prometedores para el tratamiento de la DM tipo 2 en estudios clínicos iniciales, no deben realizarse fuera de protocolos de investigación aprobados por comités de ética. Sí

Consenso: Alto

Antes de la presentación:

Votación total: Sí 93\% No 7\%

Cirujanos: $\quad$ Sí 100\% No 0\%

No cirujanos: Sí $67 \%$ No $33 \%$

Después de la presentación:

Votación total: Sí 95\% No 5\%

Cirujanos: $\quad$ Sí 100\% No 0\%

No cirujanos: Sí $75 \% \quad$ No $25 \%$

Comentario: Hubo consenso sobre la forma en que estos nuevos procedimientos deben ser investigados.

21. Pacientes con IMC $>35$ que son candidatos y que son sometidos a cirugía gastrointestinal (derivación gástrica, gástrico anillado, BPD, manga) tienen una tasa mayor de supervivencia, con disminución del riesgo $\mathrm{CV}$, comparados con los individuos que no son operados. Sí Consenso: Alto

Antes de la presentación:

Votación total: Sí $83 \% \quad$ No 17\%

Cirujanos: $\quad$ Sí 100\% No 0\%

No cirujanos: Sí 33\% No 67\%

Después de la presentación: 
Votación total: Sí 91\% No 9\%

Cirujanos: $\quad$ Sí $92 \%$ No $8 \%$

No cirujanos: Sí 100\% No 0\%

Comentario: Antes de la presentación, los no cirujanos no se dieron cuenta del impacto en el riesgo cardiovascular y supervivencia tras la cirugía bariátrica. Después de la presentación, cambiaron sensiblemente de opinión.

22. El mejor tratamiento médico no quirúrgico, incluyendo cambios de estilo de vida, dieta, ejercicio, medicamentos, etcétera, reduce la enfermedad cardiovascular (ataque al corazón y derrame cerebral) y muerte en sujetos con obesidad tipo de DM 2. Sí Consenso: No

Antes de la presentación:

Votación total: Sí 63\%

Cirujanos: $\quad$ Sí 63\%

No $38 \%$

No cirujanos: $\quad$ Sí 75\%

Después de la presentación:

Votación total: Sí 63\%

Cirujanos: $\quad$ Sí $57 \%$

No cirujanos: Sí 67\% No 33\%

No $38 \%$

No $25 \%$

Comentario: Ambos grupos están de acuerdo en que el tratamiento médico para las personas con diabetes y obesidad repercute en el riesgo cardiovascular y muerte.

23. La cirugía metabólica es el único tratamiento disponible en este momento que promueve una reducción sustancial en la enfermedad cardiovascular (ataque al corazón y derrame cerebral) y muerte en pacientes con obesidad y DM2. Sí

Consenso: Bajo

Antes de la presentación:

$\begin{array}{lll}\text { Votación total: } & \text { Sí 56\% } & \text { No 44\% } \\ \text { Cirujanos: } & \text { Sí 50\% } & \text { No 50\% } \\ \text { No cirujanos: } & \text { Sí 50\% } & \text { No 50\% }\end{array}$

Después de la presentación:

Votación total: Sí 67\% No 33\%

Cirujanos: $\quad$ Sí $64 \%$ No $36 \%$

No cirujanos: Sí 67\% No 33\%

Comentario: Aunque con bajo consenso, tanto cirujanos como no cirujanos estuvieron de acuerdo en que la cirugía metabólica tiene un impacto en la reducción de enfermedad cardiovascular y muerte en individuos diabéticos obesos.

\section{MÓDULO 4}

Mecanismos de control de la diabetes con cirugía.
24. Los procedimientos de derivación intestinal (derivación gástrica y DBP) pueden mejorar la diabetes a través de mecanismos diferentes a los cambios en alimentación y pérdida de peso. Sí

Consenso: Alto

Antes de la presentación:

Votación total: Sí 100\% No 0\%

Cirujanos: $\quad$ Sí 100\% No 0\%

No cirujanos: Sí 100\% No 0\%

Después de la presentación:

Votación total: Sí 100\% No 0\%

Cirujanos: $\quad$ Sí 100\% No 0\%

No cirujanos: Sí 100\% No 0\%

Comentario: Todos coinciden en que hay algo diferente a los cambios de alimentación y pérdida de peso para explicar la mejora de la diabetes después de procedimientos bariátricos derivativos.

25. La banda gástrica puede mejorar la diabetes a través de mecanismos diferentes que los cambios en la ingesta de alimentos y pérdida de peso. No

Consenso: Alto

Antes de la presentación:

Votación total: Sí 10\% No 90\%

Cirujanos: $\quad$ Sí $0 \% \quad$ No $100 \%$

No cirujanos: Sí 67\% No 33\%

Después de la presentación:

Votación total: Sí: 10\% No 90\%

Cirujanos: $\quad$ Sí 0\% $\quad$ No $100 \%$

No cirujanos: Sí $67 \%$ No $33 \%$

Comentario: Casi ningún cirujano parece considerar que la banda tiene un efecto metabólico. No así los no cirujanos, que están de acuerdo con un consenso bajo.

26. La efectividad clínica de la cirugía gastrointestinal (derivación gástrica, banda gástrica, BPD, manga) en DM2, enfermedades metabólicas y obesidad, indica que el tubo digestivo es un objetivo para dirigir la investigación y para desarrollar nuevos y mejores tratamientos médicos e intervencionistas. Sí Consenso: Alto

Antes de la presentación:

Votación total: Sí 100\% No 0\%

Cirujanos: $\quad$ Sí $100 \%$ No $0 \%$

No cirujanos: Sí 100\% No 0\%

Después de la presentación:

Votación total: Sí 100\% No 0\%

Cirujanos: $\quad$ Sí 100\% No 0\%

No cirujanos: $\quad$ Sí $100 \%$ No 0\% 
Comentario: Hay un acuerdo en el concepto de que el tubo digestivo está relacionado con la explicación de los cambios metabólicos positivos en la DM2, síndrome metabólico y obesidad después de la cirugía.

\section{MÓDULO 5}

Investigación.

27. En un sujeto con un IMC $<35$, determinar el uso apropiado de la cirugía gastrointestinal para tratamiento de la DM2 es una prioridad en la investigación. Sí

Consenso: Alto

Antes de la presentación:

Votación total: Sí 100\% No 0\%

Cirujanos: $\quad$ Sí 100\% No 0\%

No cirujanos: Sí 100\% No 0\%

Después de la presentación:

Votación total: Sí 100\% No 0\%

Cirujanos: $\quad$ Sí 100\% No 0\%

No cirujanos: Sí 100\% No 0\%

28. Se debe promover la realización de estudios clínicos controlados para conocer el papel de la cirugía gastrointestinal en el tratamiento de personas con un IMC $<35$ con DM2 que son controladas inadecuadamente por estilo de vida y tratamiento médico. Sí

Consenso: Alto

Antes de la presentación:

Votación total: Sí 100\% No 0\%

Cirujanos: $\quad$ Sí 100\% No 0\%

No cirujanos: Sí 100\% No 0\%

Después de la presentación:

Votación total: Sí 100\% No 0\%

Cirujanos: $\quad$ Sí 100\% No 0\%

No cirujanos: Sí 100\% No 0\%

29. Es necesario establecer un sistema unificado de informe de los resultados clínicos en cirugía bariátrica y metabólica.

Consenso: Alto

Antes de la presentación:

Votación total: Sí 100\%

Cirujanos: $\quad$ Sí 100\%

No cirujanos: Sí 100\%

Después de la presentación:

Votación total: Sí 100\%

Cirujanos: $\quad$ Sí 100\%

\section{MÓDULO 6}

Creación de la fuerza de tarea para el estudio de la cirugía metabólica en México (voto sin presentación).

30. Estoy de acuerdo en que la cirugía bariátrica es un componente de la cirugía metabólica. Sí

Consenso: Medio

Votación total: Sí $82 \% \quad$ No $18 \%$

Cirujanos: $\quad$ Sí 100\% No 0\%

No cirujanos: Sí 50\% No 50\%

Comentario: Aunque existe un consenso sobre este concepto, los no cirujanos están divididos en sus opiniones.

31. Es importante trabajar juntos, los clínicos y los cirujanos, para desarrollar programas de excelencia para el uso de la cirugía metabólica. Sí

\section{Consenso: Alto}

$\begin{array}{lll}\text { Votación total: } & \text { Sí 100\% } & \text { No 0\% } \\ \text { Cirujanos: } & \text { Sí 100\% } & \text { No 0\% } \\ \text { No cirujanos: } & \text { Sí 100\% } & \text { No 0\% }\end{array}$

32. Mejorar el acceso de los pacientes a la cirugía bariátrica y metabólica en México es una prioridad. Sí

Consenso: Medio

$\begin{array}{lll}\text { Votación total: } & \text { Sí 88\% } & \text { No 12\% } \\ \text { Cirujanos: } & \text { Sí 100\% } & \text { No 0\% } \\ \text { No cirujanos: } & \text { Sí } 67 \% & \text { No 33\% }\end{array}$

33. La cirugía metabólica debería estudiarse de manera conjunta por los clínicos (endocrinólogos, diabetólogos, internistas, cardiólogos, etcétera) y cirujanos (bariátricos y metabólicos). Sí

Consenso: Alto
Votación total:
Cirujanos:
Sí 100\%
No $0 \%$
No cirujanos: $\quad$ Sí 100\% No 0\%

34. Es necesaria una fuerza de trabajo en México para el estudio de cirugía metabólica. Sí Consenso: Alto
Votación total:
Cirujanos:
Sí 100\%
No $0 \%$
Sí 100\% No 0\%
No cirujanos: Sí 100\% No 0\%

35. Una ventaja de contar con una fuerza de tarea en México para el estudio de la cirugía 
metabólica es promover el diálogo científico entre los médicos y cirujanos. Sí

Consenso: Alto

$\begin{array}{lll}\text { Votación total: } & \text { Sí 100\% } & \text { No 0\% } \\ \text { Cirujanos: } & \text { Sí 100\% } & \text { No 0\% } \\ \text { No cirujanos: } & \text { Sí 100\% } & \text { No 0\% }\end{array}$

36. Una ventaja de contar con una fuerza de tarea en México para el estudio de la cirugía metabólica es facilitar el diálogo entre las asociaciones clínicas y quirúrgicas. Sí Consenso: Alto
Votación total:
Cirujanos:
Sí 100\%
No $0 \%$
No cirujanos:
Sí 100\%
No $0 \%$
No $0 \%$

37. Una ventaja de tener una fuerza de tarea en México para el estudio de la cirugía metabólica es ayudar a definir objetivos comunes. Sí

Consenso: Alto

$\begin{array}{lll}\text { Votación total: } & \text { Sí 100\% } & \text { No 0\% } \\ \text { Cirujanos: } & \text { Sí 100\% } & \text { No 0\% } \\ \text { No cirujanos: } & \text { Sí 100\% } & \text { No 0\% }\end{array}$

38. Una ventaja de tener una fuerza de tarea en México para el estudio de la cirugía metabólica es evaluar el avance en la consecución de los objetivos. Sí

Consenso: Alto

$\begin{array}{lll}\text { Votación total: } & \text { Sí 100\% } & \text { No 0\% } \\ \text { Cirujanos: } & \text { Sí 100\% } & \text { No 0\% } \\ \text { No cirujanos: } & \text { Sí 100\% } & \text { No 0\% }\end{array}$

39. Una ventaja de tener una fuerza de tarea en México para el estudio de la cirugía metabólica es que el trabajo puede programarse. Sí Consenso: Alto
Votación total:
Cirujanos:
Sí $95 \%$
Sí 88\%
No $5 \%$
No cirujanos:
Sí 100\%
No $12 \%$
No $0 \%$

40. Una ventaja de tener una fuerza de tarea en México para el estudio de la cirugía metabólica es promover y organizar el trabajo de investigación. Sí

Consenso: Alto
Votación total:
Cirujanos:
Sí $94 \%$
Sí $86 \%$
No $5 \%$
No cirujanos:
Sí 100\%
No $14 \%$
No $0 \%$

41. Una ventaja de tener una fuerza de tarea en México para el estudio de la cirugía metabólica es favorecer la obtención de patrocinios para la investigación. Sí

Consenso: Medio

$\begin{array}{lll}\text { Votación total: } & \text { Sí } 89 \% & \text { No } 11 \% \\ \text { Cirujanos: } & \text { Sí } 80 \% & \text { No } 20 \% \\ \text { No cirujanos: } & \text { Sí } 90 \% & \text { No } 10 \%\end{array}$

42. Una ventaja de tener una fuerza de tarea en México para el estudio de la cirugía metabólica es mantener un estrecho contacto con la fuerza de tarea internacional para promover la propagación de conocimientos sobre cirugía metabólica. Sí

Consenso: Alto
Votación total:
Cirujanos:
Sí 95\%
No $6 \%$
No cirujanos:
Sí $88 \%$
No $12 \%$
Sí $100 \%$ No 0\%

43. Un propósito para esta fuerza de tarea es difundir con veracidad el conocimiento acerca de la cirugía metabólica. Sí

Consenso: Alto
Votación total:
Cirujanos:

$\begin{array}{ll}\text { Sí: } 95 \% & \text { No } 5 \% \\ \text { Sí } 88 \% & \text { No } 12 \% \\ \text { Sí } 100 \% & \text { No } 0 \%\end{array}$

44. Un propósito para esta fuerza de tarea es alertar sobre el uso indebido de la cirugía metabólica en nuestro país. Sí

Consenso: Medio

$\begin{array}{lll}\text { Votación total: } & \text { Sí } 86 \% & \text { No 14\% } \\ \text { Cirujanos: } & \text { Sí } 78 \% & \text { No 22\% } \\ \text { No cirujanos: } & \text { Sí 100\% } & \text { No 0\% }\end{array}$

45. Un propósito para esta fuerza de tarea es publicar recomendaciones sobre el uso y aplicación de la cirugía metabólica. Sí Consenso: Alto
Votación total:
Cirujanos:
Sí 95\%
No $5 \%$
No cirujanos:
Sí 100\%
No $11 \%$
No $0 \%$

46. Un propósito para esta fuerza de tarea es mantener el diálogo científico entre los médicos y cirujanos que estudian y realizan la cirugía metabólica. Sí

Consenso: Alto
Votación total:
Sí $94 \%$
Cirujanos: $\quad$ Sí $86 \%$
No $6 \%$
No cirujanos:
Sí 100\%
No $14 \%$
No $0 \%$

47. Un propósito para esta fuerza de tarea es asesorar a sociedades médicas y quirúrgicas 
para ayudarlas a elaborar posicionamientos conjuntos sobre cirugía metabólica. Sí

Consenso: Alto

$\begin{array}{lll}\text { Votación total: } & \text { Sí: } 100 \% & \text { No 0\% } \\ \text { Cirujanos: } & \text { Sí 100\% } & \text { No 0\% } \\ \text { No cirujanos: } & \text { Sí 100\% } & \text { No 0\% }\end{array}$

48. Un propósito para esta fuerza de tarea es mejorar el acceso a la cirugía bariátrica y metabólica. Sí

Consenso: Alto

Votación total: Sí 100\% No 0\%

Cirujanos: $\quad$ Sí 100\% No 0\%

No cirujanos: Sí 100\% No 0\%

49. ¿Quién debe formar parte de esta fuerza de tarea? Miembros señalados por sus sociedades clínicas o quirúrgicas. Sí

Consenso: Bajo

$\begin{array}{lll}\text { Votación total: } & \text { Sí 72\% } & \text { No 28\% } \\ \text { Cirujanos: } & \text { Sí 100\% } & \text { No 0\% } \\ \text { No cirujanos: } & \text { Sí } 67 \% & \text { No 33\% }\end{array}$

50. ¿Quién debe formar parte de esta fuerza de tarea? Expertos en endocrinología, diabetes, cirugía bariátrica y metabólica. Sí

Consenso: Alto

$\begin{array}{lll}\text { Votación total: } & \text { Sí 100\% } & \text { No 0\% } \\ \text { Cirujanos: } & \text { Sí 100\% } & \text { No 0\% } \\ \text { No cirujanos: } & \text { Sí 100\% } & \text { No 0\% }\end{array}$

51. ¿Quién debe formar parte de esta fuerza de tarea? Expertos escogidos por la Secretaría de Salud. No

Consenso: Alto

$\begin{array}{lll}\text { Votación total: } & \text { Sí: 10\% } & \text { No } 90 \% \\ \text { Cirujanos: } & \text { Sí 22\% } & \text { No } 78 \% \\ \text { No cirujanos: } & \text { Sí 0\% } & \text { No 100\% }\end{array}$

52. ¿Quién debe formar parte de esta fuerza de tarea? El grupo para el estudio del síndrome metabólico. No

Consenso: No

$\begin{array}{lll}\text { Votación total: } & \text { Sí } 53 \% & \text { No } 47 \% \\ \text { Cirujanos: } & \text { Sí } 75 \% & \text { No } 25 \% \\ \text { No cirujanos: } & \text { Sí } 40 \% & \text { No } 60 \%\end{array}$

53. ¿Quién puede patrocinar a este equipo de trabajo? Sociedades médicas y quirúrgicas. Sí Consenso: Bajo

$\begin{array}{lll}\text { Votación total: } & \text { Sí: } 91 \% & \text { No } 9 \% \\ \text { Cirujanos: } & \text { Sí 100\% } & \text { No } 0 \% \\ \text { No cirujanos: } & \text { Sí } 83 \% & \text { No } 17 \%\end{array}$

54. ¿Quién puede patrocinar a este equipo de trabajo? La Secretaría de Salud. Sí

Consenso: Alto

$\begin{array}{lll}\text { Votación total: } & \text { Sí: } 93 \% & \text { No 7\% } \\ \text { Cirujanos: } & \text { Sí 100\% } & \text { No 0\% } \\ \text { No cirujanos: } & \text { Sí } 83 \% & \text { No 17\% }\end{array}$

55. ¿Quién puede patrocinar a este equipo de trabajo? La industria médica y farmacéutica. Sí

Consenso: Alto

$\begin{array}{lll}\text { Votación total: } & \text { Sí 89\% } & \text { No 11\% } \\ \text { Cirujanos: } & \text { Sí 100\% } & \text { No 0\% } \\ \text { No cirujanos: } & \text { Sí } 80 \% & \text { No } 20 \%\end{array}$

56. ¿Quién puede patrocinar a este equipo de trabajo? La industria quirúrgica. Sí Consenso: Medio

Votación total: Sí 89\% No 11\%

Cirujanos: $\quad$ Sí $100 \%$ No 0\%

No cirujanos: $\quad$ Sí $80 \% \quad$ No $20 \%$

57. ¿Las condiciones son favorables en este foro para crear esta fuerza de tarea? Sí

Consenso: No

Votación total: Sí 63\% No 38\%

Cirujanos: $\quad$ Sí $71 \%$ No $29 \%$

No cirujanos: Sí 25\% No $75 \%$

Comentario: Los no cirujanos consideraron que las condiciones no eran favorables para crear la fuerza de tarea.

58. ¿Debe realizarse un tercer foro para el estudio de cirugía metabólica en México? Sí Consenso: Medio

$\begin{array}{lll}\text { Votación total: } & \text { Sí } 86 \% & \text { No 14\% } \\ \text { Cirujanos: } & \text { Sí 89\% } & \text { No 11\% } \\ \text { No cirujanos: } & \text { Sí 100\% } & \text { No 0\% }\end{array}$

\section{MÓDULO 7}

Revisión de grados de consenso durante el foro. Disposición de las asociaciones médicas y quirúrgicas que participaron en el foro para crear posicionamientos sobre cirugía metabólica (voto sin presentación).

59. ¿Por qué es importante hacer un posicionamiento sobre cirugía metabólica en México? Porque da claridad en las indicaciones para la cirugía metabólica en nuestro país. Sí

Consenso: Alto 
Votación total: Sí: 100\% No 0\%

Cirujanos: $\quad$ Sí $100 \%$ No 0\%

No cirujanos: Sí 100\% No 0\%

Comentario: Sociedades y asociaciones consideran que es importante elaborar un posicionamiento sobre la cirugía metabólica.

60. ¿Por qué es importante hacer un posicionamiento sobre cirugía metabólica en México? Porque define lo que es el uso inadecuado de cirugía metabólica en México. Sí Consenso: Alto

$\begin{array}{lll}\text { Votación total: } & \text { Sí 100\% } & \text { No 0\% } \\ \text { Cirujanos: } & \text { Sí 100\% } & \text { No 0\% } \\ \text { No cirujanos: } & \text { Sí 100\% } & \text { No 0\% }\end{array}$

61. ¿Por qué es importante hacer un posicionamiento sobre cirugía metabólica en México? Porque orienta el trabajo clínico diario. Sí Consenso: Alto

$\begin{array}{lll}\text { Votación total: } & \text { Sí 86\% } & \text { No 14\% } \\ \text { Cirujanos: } & \text { Sí 80\% } & \text { No 20\% } \\ \text { No cirujanos: } & \text { Sí 100\% } & \text { No 0\% }\end{array}$

62. ¿Por qué es importante hacer un posicionamiento sobre cirugía metabólica en México? Porque mejora la comunicación entre clínicos y cirujanos. Sí

Consenso: Alto

$\begin{array}{lll}\text { Votación total: } & \text { Sí 93\% } & \text { No 7\% } \\ \text { Cirujanos: } & \text { Sí } 90 \% & \text { No 10\% } \\ \text { No cirujanos: } & \text { Sí 100\% } & \text { No 0\% }\end{array}$

63. ¿Por qué es importante hacer un posicionamiento sobre cirugía metabólica en México? Porque ayuda a elaborar líneas de investigación sobre cirugía metabólica. Sí Consenso: Alto
Votación total:
Cirujanos:
Sí 94\%
Sí 83\%
No $6 \%$
No cirujanos:
Sí 100\%
No $17 \%$
No $0 \%$

64. ¿Por qué es importante hacer un posicionamiento sobre cirugía metabólica en México? Porque facilita el intercambio científico entre la comunidad médica nacional e internacional. Sí

Consenso: Alto

$\begin{array}{lll}\text { Votación total: } & \text { Sí 100\% } & \text { No 0\% } \\ \text { Cirujanos: } & \text { Sí 100\% } & \text { No 0\% } \\ \text { No cirujanos: } & \text { Sí 100\% } & \text { No 0\% }\end{array}$

65. ¿La sociedad, asociación, colegio que represento estaría interesado en realizar un posicionamiento sobre cirugía metabólica en México? Sí

Consenso: Alto

$$
\begin{array}{lll}
\text { Votación total: } & \text { Sí 100\% } & \text { No 0\% } \\
\text { Cirujanos: } & \text { Sí 100\% } & \text { No 0\% } \\
\text { No cirujanos: } & \text { Sí 100\% } & \text { No 0\% }
\end{array}
$$

66. ¿Tomaría yo los grados de consenso que obtuvimos en este foro junto con las guías internacionales como elementos para la sociedad, asociación, colegio que yo represento para elaborar un posicionamiento sobre cirugía metabólica en México? Sí

Consenso: Bajo

$\begin{array}{lll}\text { Votación total: } & \text { Sí 89\% } & \text { No 11\% } \\ \text { Cirujanos: } & \text { Sí 67\% } & \text { No 33\% } \\ \text { No cirujanos: } & \text { Sí 100\% } & \text { No 0\% }\end{array}$

67. Tomando en cuenta los grados de consenso que se obtuvieron durante este foro, itengo suficientes elementos de juicio para dar a la sociedad, asociación, colegio o institución que represento para crear un posicionamiento sobre cirugía metabólica en México? Sí

Consenso: Medio

$\begin{array}{lll}\text { Votación total: } & \text { Sí } 80 \% & \text { No 20\% } \\ \text { Cirujanos: } & \text { Sí } 75 \% & \text { No 25\% } \\ \text { No cirujanos: } & \text { Sí 100\% } & \text { No 0\% }\end{array}$

68. La sociedad, asociación, colegio que yo represento, iestá interesado en conocer otros posicionamientos sobre la cirugía metabólica en México?

Consenso: Alto
Votación total:
Cirujanos:
Sí 100\%
No $0 \%$
Sí $100 \%$ No 0\%
No cirujanos: $\quad$ Sí 100\% No 0\%

69. Como presidente de la sociedad, asociación, colegio que represento, ime comprometo a transmitir el documento que será escrito en este foro entre los miembros de mi asociación?

Consenso: Alto

$\begin{array}{lll}\text { Votación total: } & \text { Sí 100\% } & \text { No 0\% } \\ \text { Cirujanos: } & \text { Sí 100\% } & \text { No 0\% } \\ \text { No cirujanos: } & \text { Sí 100\% } & \text { No 0\% }\end{array}$

